\title{
BP-OP-3-5
}

\section{Optimal timing of surgical resection after neoadjuvant chemoradiation in borderline and locally advanced pancreatic ductal adenocarcinoma}

\author{
Munseok $\mathrm{CHOI}^{1}$, Sung Hyun $\mathrm{KIM}^{2,3}$, Ho Kyoung HWANG ${ }^{2,3}$, Woo Jung LEE ${ }^{2,3}$, Chang Moo KANG*2,3 \\ 'Department of Surgery, Yongin Severance Hospital, Yonsei University College of Medicine, Yongin, Korea \\ ${ }^{2}$ Division of Hepatobiliary and Pancreatic Surgery, Department of Surgery, Yonsei University College of Medicine, Seoul, Korea \\ ${ }^{3}$ Pancreaticobiliary Cancer Clinic, Yonsei Cancer Center, Severance Hospital, Seoul, Korea
}

Introduction: For patients with pancreatic ductal adenocarcinoma (PDAC), the optimal time interval between neoadjuvant chemoradiation to surgical resection has not been well established. We aimed to study the impact of optimal surgical resection timing after neoadjuvant chemoradiation (Neo-CT/RT) on long-term oncological outcome in borderline and locally advanced PDAC.

Methods: From June 2006 to April 2019, among the 175 patients who underwent pancreatectomy following Neo-CT/RT, the study enrolled 79 patients who underwent surgical resection after Neo-CT/RT for borderline and locally advanced PDAC. The entire cohort was divided into three groups according to the time interval between the last date or neoadjuvant chemoradiation and the surgical resection; group A ( $0-5$ weeks), group B (5-10 weeks), and group C (> 10 weeks). Long-term outcomes were compared between the three groups.

Results: There were 30 patients in group A, 37 patients in group B, and 12 patients in group C. Patients in group B were significantly more likely to experience a vascular resection than group A and C $(p=0.005)$. In disease-free survival, there was no significant difference between the three groups $(p=0.075)$. Patients in group B showed better overall survival than other groups $(p=0.041)$. In multivariate analysis, N2 stage and time interval more than ten weeks were correlated with worse overall survival (HR 7.935, 95\% confidence interval [CI] 1.772-35.538, $p=0.007$, HR 3.492, 95\% CI 1.717-7.102, $p=0.001$ ).

Conclusions: For borderline and locally advanced PDAC, surgical resection within ten weeks from the end of the Neo-CT/RT is associated with better overall survival, suggesting time interval should be concerned for better oncologic outcome in a patient with Neo$\mathrm{CT} / \mathrm{RT}$ in borderline and locally advanced PDAC. Further study is mandatory. 